\title{
Duplication at chromosome 2q31.1-q31.2 in a family presenting syndactyly and nystagmus
}

\author{
Jamal Ghoumid ${ }^{1}$, Joris Andrieux ${ }^{2}$, Bernard Sablonnière ${ }^{1}$, Sylvie Odent ${ }^{3}$, Nathalie Philippe ${ }^{1}$, Xavier Zanlonghi ${ }^{4}$, \\ Pascale Saugier-Veber ${ }^{5}$, Thomas Bardyn ${ }^{1}$, Sylvie Manouvrier-Hanu ${ }^{6}$ and Muriel Holder-Espinasse ${ }^{\star, 6}$
}

HOXD genes encode transcription factors involved in the antero-posterior patterning of the limb bud and in the specification of fingers. During the embryo development, HOXD genes are expressed, following a spatio-temporal colinearity that involves at least three regions, centrometric and telomeric to this cluster. Here, we describe a father and a daughter presenting a 3-4 hand bilateral syndactyly associated with a nystagmus. Array-comparative genomic hybridisation showed a $3.8 \mathrm{Mb}$ duplication at 2q31.1-q31.2, comprising 27 genes including the entire HOXD cluster. We performed expression studies in lymphoblasts by reverse transcription-PCR and observed an HOXD13 and HOXD10 overexpression, whereas the HOXD12 expression was decreased. HOXD13 and HOXD10 overexpression, associated with a misregulation of at least HOXD12, may therefore induce the syndactyly. Deletions of the HOXD cluster and its regulatory sequences induce hand malformations and, particularly, finger anomalies. Recently, smaller duplications of the same region have been reported in association with a mesomelic dysplasia, type Kantaputra. We discuss the variable phenotypes associated with such $2 q$ duplications.

European Journal of Human Genetics (2011) 19, 1198-1201; doi:10.1038/ejhg.2011.95; published online 8 June 2011

Keywords: syndactyly; HOXD cluster; 2q31.1q31.2 duplication

\section{INTRODUCTION}

HOXD genes encode a family of highly conserved transcription factors involved in the antero-posterior patterning of the limb bud and in the specification of fingers. ${ }^{1,2}$ During embryo development, HOXD genes are expressed following a spatio-temporal colinearity involving at least three regulatory regions, centrometric (ELCR) and telomeric (POST and Global Central Region (GCR)-Prox) to the cluster. ${ }^{3}$ Moreover, these genes are expressed through two waves $^{4}$ during the limb budding, and control the patterning of the stylopod and the zeugopod. ${ }^{5}$ The width and the efficiency of the genes' expression depend on their rank in the cluster. Each gene presents a precise pattern of expression. HOXD13, the most $5^{\prime}$ end located gene, is highly expressed throughout the presumptive digits, whereas HOXD10, HOXD11 and HOXD12 are restricted to presumptive digit 2-5 and are underexpressed. In man, deletions of this cluster induce hand malformations and particularly finger anomalies. ${ }^{5}$ Deletions of the whole cluster can cause severe defects, whereas deletions removing only HOXD9HOXD13 are responsible for a milder phenotype including fifth finger clinodactyly, variable cutaneous syndactyly of toes, hypoplastic middle phalanges of the feet and synpolydactyly. ${ }^{5,6}$ Deletions removing GCR are deleterious too, but induce minor anomalies. ${ }^{7-9}$ ELCR has not been localised so far. Its role is so critical that deletions would be lethal and thus there is no animal model.

Animal models carrying internal duplications of part of the HOXD cluster and limb anomalies exist. ${ }^{3,4}$ Indeed, mice with targeted disruptions of Hoxd11 and Hoxa11 genes showed marked zeugopod malformation. ${ }^{10}$ A disconnection of $5^{\prime}$ Hoxd genes from the regulator could result in a downregulation of $5^{\prime}$ Hoxd genes in the distal limb (autopod) and an upregulation in the proximal limb, and it has been suggested that the 2q duplication could have the same effect, therefore, explaining the mesomelic dysplasia recently reported. ${ }^{11,4}$ Indeed, it has been recently reported that a $1 \mathrm{Mb}$ microduplication of HOXD gene cluster at $2 \mathrm{q} 31.1$ is associated with a dominant mesomelic dysplasia, Kantaputra type. The condition is mainly affecting the upper limbs and is very variable among affected patients within the same family. This phenotype, linked with a small $2 \mathrm{q}$ duplication that contain the entire HOXD cluster, is far more severe than the one we report here, in which the duplicaton is larger and involving several other genes.

Indeed, we report on a father and his daughter referred to the genetic clinic for the association of bilateral 3-4 finger cutaneous syndactyly and nystagmus carrying a 2q31.1q31.2 duplication involving 27 genes, among which the whole HOXD cluster, identified by array-comparative genomic hybridisation (CGH). We characterise this chromosomal anomaly and discuss the genotype-phenotype correlations.

\section{PATIENTS AND METHODS}

The probands are a father and his daughter. The father presents an association of bilateral 3-4 hand cutaneous syndactyly and a pendular-resilient nystagmus, which increases in up and right gaze and decreases in down gaze. Ophthalmologic examination and functional tests were normal (slit-lamp, fundoscopy, binocular visual field and electroretinogram), as well as a full neurological examination. Skeletal survey including hand X-rays was normal (Figure 1a). His 6-year-old daughter is affected with the same hand malformations (Figure 1b). She was born after an uneventful pregnancy. Motor milestones were achieved normally and her psychomotor development is in correlation

${ }^{1}$ Department of Molecular Biology, University Hospital, Lille, France; ${ }^{2}$ Department of Cytogenetics, University Hospital, Lille, France; ${ }^{3}$ Department of Clinical Genetics, University Hospital, Rennes, France; ${ }^{4}$ Department of Ophthalmology, University Hospital, Nantes, France; ${ }^{5}$ Department of Molecular Biology, University Hospital, Rouen, France; ${ }^{6}$ Department of Clinical Genetics, University Hospital, Lille, France

*Correspondence: Dr M Holder-Espinasse, Service de Génétique Clinique, Hôpital Jeanne de Flandre, CHRU, 59037 Lille Cedex, France. Tel: +003 3320444911 ; Fax: +003 332044 4901; E-mail: muriel.holder@chru-lille.fr

Received 20 July 2010; revised 7 April 2011; accepted 27 April 2011; published online 8 June 2011 


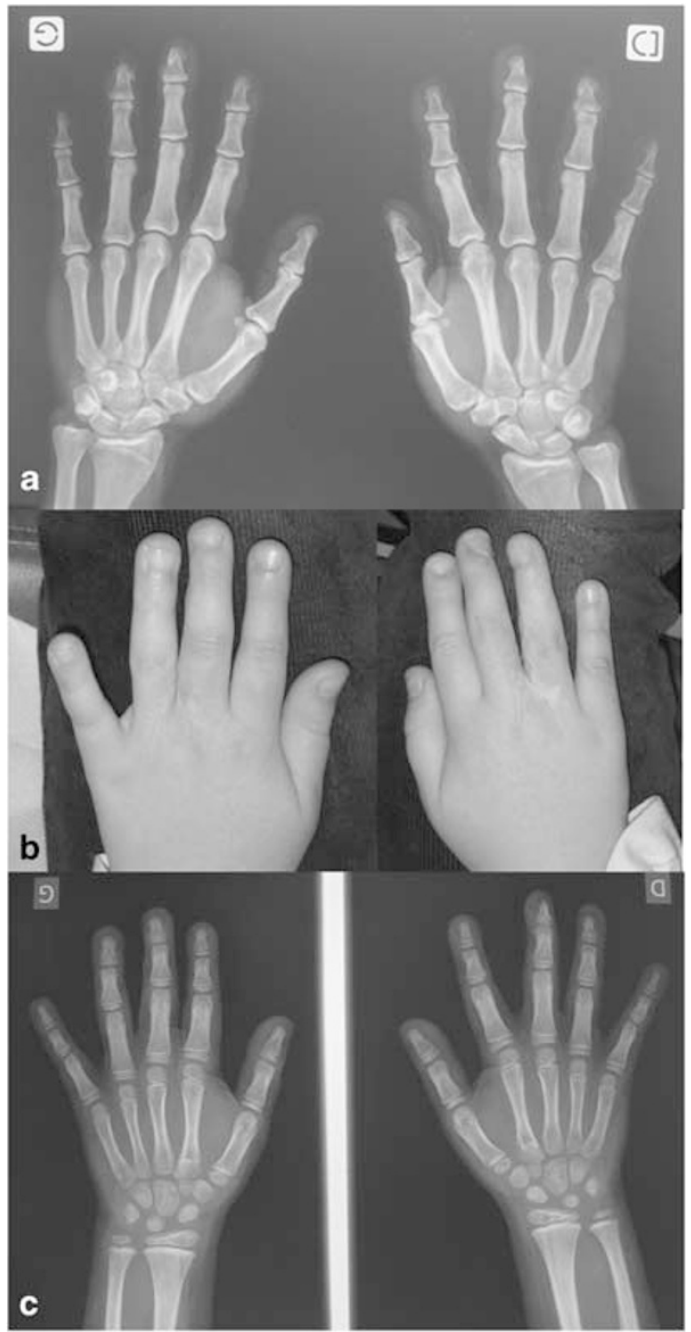

Figure 1 (a) Normal hands X-rays of the affected father. (b) Hands of the daughter after surgery. Note scars after surgery for 3-4 fingers' skin syndactyly. (c) Normal hands X-rays of the affected daughter.

with her age. Her growth parameters are advanced $(123 \mathrm{~cm},+2.5 \mathrm{SD}$ for the height; $29 \mathrm{~kg},+3 \mathrm{SD}$ for the weight; $51 \mathrm{~cm},-0.5 \mathrm{SD}$ for the head circumference). No dysmorphic features were identified. She presents a slow pendular nystagmus, which causes amblyopy (confirmed by visual evoked potentials). A full neurological examination was normal, as well as a skeletal survey including hand $\mathrm{X}$-rays (Figure 1c).

Genomic DNA was extracted from peripheral blood lymphocytes for both patients and the unaffected mother. Detection of gene copy number was performed by array-CGH (Agilent, Agilent Technologies, Santa Clara, CA, USA) using 44000 oligo probes, approximately spaced at $40-100 \mathrm{~kb}$ intervals across the genome (Human Genome CGH microarray 44B kit, Agilent). Male and female genomic DNAs (Promega, Madison, WI, USA) were used as reference in hybridisations, which were analysed with the CGH-analytics software by applying a Z-score segmentation algorithm to identify chromosome aberrations.

Quantitative-PCR was performed on genomic DNA extracted from the three members of the family. TaqMan analyses were performed in Fast Gene Quantification (Applied Biosystems, Villebon-sur-Yvette, France) in 96-well plates. The final volume was $20 \mu \mathrm{l}$ and contained Genotyping master Mix (Applied Biosystems) (15 $\mu \mathrm{l}), 4 \mu \mathrm{l}$ of DNA and $1 \mu \mathrm{l}$ of specific genes' primers and probes. Two exons per gene were studied: HOXD13 (exon 1 and 2), HOXD12 (exon 1 and 2), HOXD10 (exon 1 and 2), CHN1 (exon 1 and 13) and CHRNA1 (exon 2 and 10). All reactions were performed in triplicate. Thermal cycling conditions were as follows: denaturation at $95^{\circ} \mathrm{C}$ for $10 \mathrm{~min}$ and 40 cycles of $95^{\circ} \mathrm{C}$ for $15 \mathrm{~s}, 60^{\circ} \mathrm{C}$ for $60 \mathrm{~s}$ and $72^{\circ} \mathrm{C}$ for $60 \mathrm{~s}$. Analyses were carried out on a 7900HT Sequence Detection System (Applied Biosystems) and interpreted with the comparative Ct methods. RNASEP was used as the reference gene control.

Total RNA was extracted from lymphoblasts cell cultures, using the RNeasy Mini Kit (Qiagen GmgH, Hilde, Germany) following the manufacturer's instructions. A total of $2 \mu \mathrm{g}$ of RNA was retrotranscripted into cDNA, with the High Capacity RNA-to-cDNA kit (Applied Biosystems, Foster City, CA, USA), for $1 \mathrm{~h}$ at $37^{\circ} \mathrm{C}$. TaqMan analyses were performed in Fast Gene Quantification in 96-well plates. The final volume was $20 \mu \mathrm{l}$ and contained Gene Expression master Mix (Applied Biosystems) $(10 \mu \mathrm{l}), 2 \mu \mathrm{l}$ of cDNA and $1 \mu \mathrm{l}$ of specific Gene Expression Assays for human HOXD13, HOXD12, HOXD10, CHN1, CHRNA1 and RPL13A (primers and probes sequences Applied Biostystems), following manufacturers' instructions. All reactions were performed in quadruplicate. Thermal cycling conditions were as follows: denaturation at $95^{\circ} \mathrm{C}$ for $10 \mathrm{~min}$ and 40 cycles of $95^{\circ} \mathrm{C}$ for $15 \mathrm{~s}, 60^{\circ} \mathrm{C}$ for $60 \mathrm{~s}$ and $72^{\circ} \mathrm{C}$ for $60 \mathrm{~s}$. Analyses were carried out on a 7900HT Sequence Detection System and interpreted with the comparative Ct methods. RPL13A was used as the reference gene control.

Chromosomal analyses of peripheral blood lymphocytes, according to routine procedures, using GTG-banding (550 bands), and FISH analyses using bacterial artificial chromosome clone RP11-483E17 localised at 2q31.1 (chr2:175,041,497-175,231,429) and clone RP11-250N10 localised at 2q31.2 (chr2:178,079,879-178,252,293) (hg18, NCBI Build 36), were performed in both patients.

\section{RESULTS}

We report on a father and his daughter presenting a congenital nystagmus and a 3-4 hand bilateral syndactyly. Array-CGH identified a $3.8 \mathrm{Mb}$-wide $2 \mathrm{q} 31.1 \mathrm{q} 31.2$ duplication, which comprises 27 genes and involves the whole HOXD cluster, CHN1 and CHRNA1, and also a large portion of local chromosome environment (Figure 2).

We studied the level of cDNA in lymphoblasts to evaluate the impact of the duplication on the involved genes' expression. Quantitative-PCR analysis confirmed the duplication of HOXD13, HOXD12, HOXD10, CHN1 and CHRNA1. The analysed exons of each tested gene were double dosed in the duplicated patients, comparatively to the unaffected mother (Figure 3). HOXD13 and HOXD10 were overexpressed in the father (3.7 and 2.9 fold, respectively) and his daughter (3.0 and 6.2 fold, respectively). The expression of HOXD12 was diminished in the daughter ( 5 fold), but in the father, no difference was shown (data not shown).

Chromosome analyses (550 bands) were normal and FISH analyses revealed direct $2 \mathrm{q} 31.1 \mathrm{q} 32.2$ duplication in both patients (Figure 4).

\section{DISCUSSION}

We report on a father and his daughter presenting a large $2 q 31.1$ duplication involving the HOXD cluster, but also many other genes, and a very mild phenotype, namely a cutaneous syndactyly between two fingers and a nystagmus. Recently, two reports on a dominant mesomelic dysplasia type Kantaputra have been described in association with a 2q31.1 duplication involving the HOXD locus and other genes (MTX2, EVX2, KIAA1715), out of which some are also known to have important roles during digit development. ${ }^{14,11}$ The patients presented severe shortening of the middle segments of the arm, relative shortening of the tibia and fibula and no ophthalmologicalassociated anomaly. As our cases had a normal full skeletal survey, their phenotype is very different and is restricted to a bilateral cutaneous syndactyly between the third and fourth finger. The $2 \mathrm{q} 31.1$ duplication in our cases was larger than that reported by the previous authors. ${ }^{14,11}$ We do not know whether our cases' phenotype is linked with increased gene expression or dysregulation at the HOXD locus. HOXD13 overexpression might explain the cutaneous syndac- 

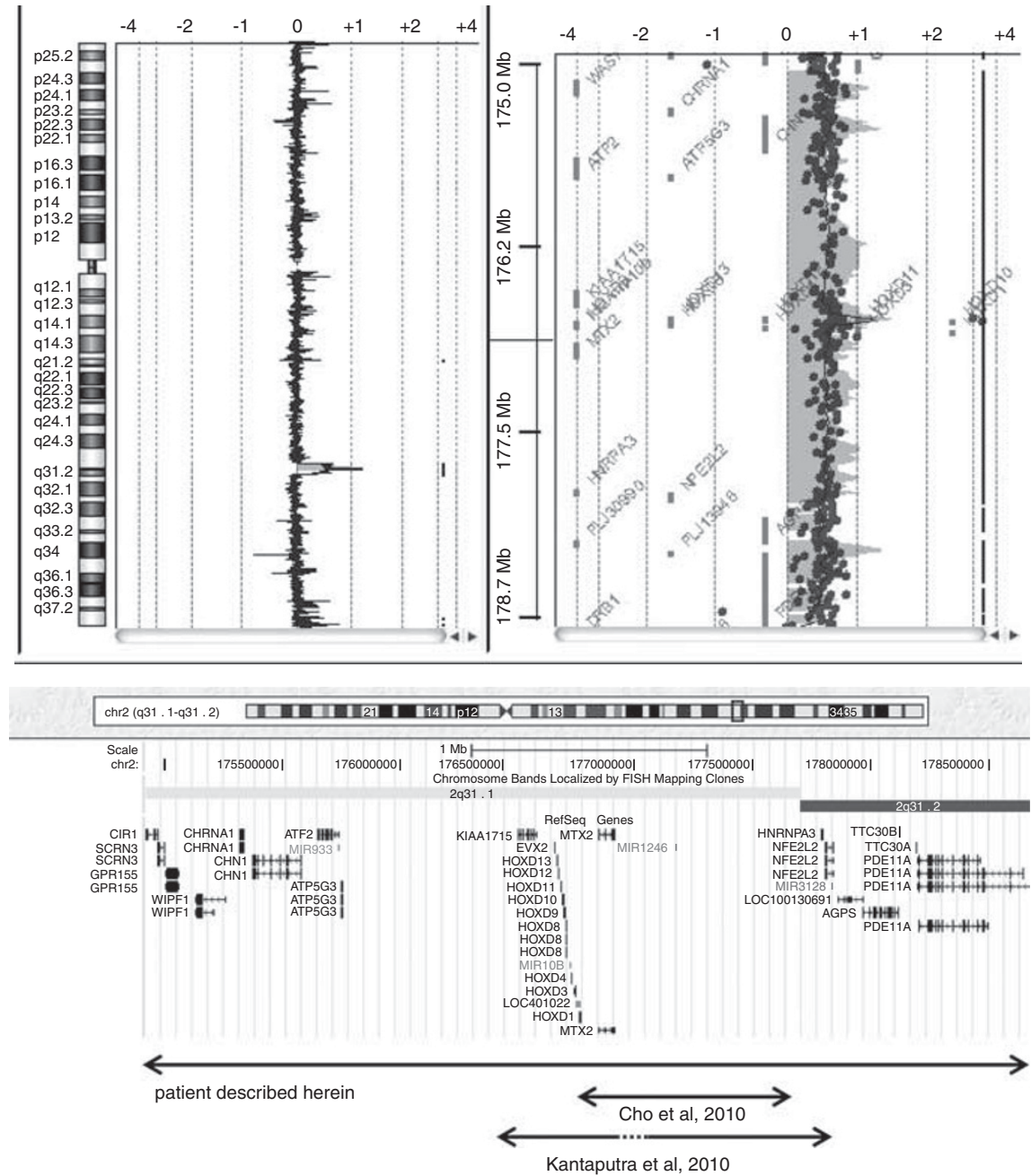

Figure 2 Array-CGH analysis and genes involved in the $3.8 \mathrm{Mb}$-wide $2 \mathrm{q} 31$ duplication. Comparison with the $2 q 31$ duplication involved in the Kantaputra mesomelic dysplasia.

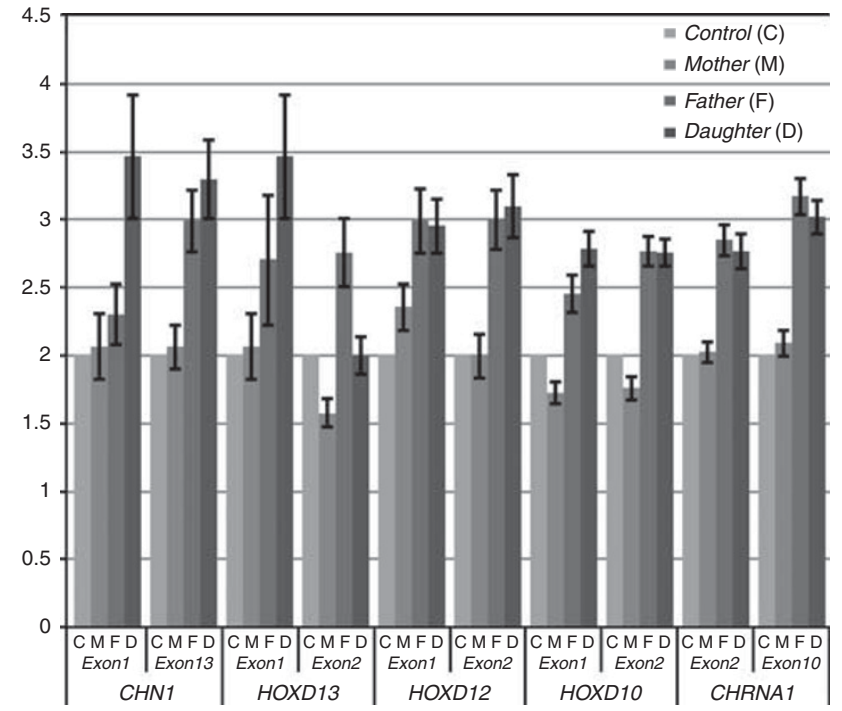

Figure 3 Quantative-PCR analysis. Estimated copy variatons for the different analysed exons of CHN1, HOXD13, HOXD12, HOXD10 and CHRNA1. The confidence interval is $95 \%$ with $n=4$. There are three copies in the father and his daughter, whereas there are two in the mother and the control.

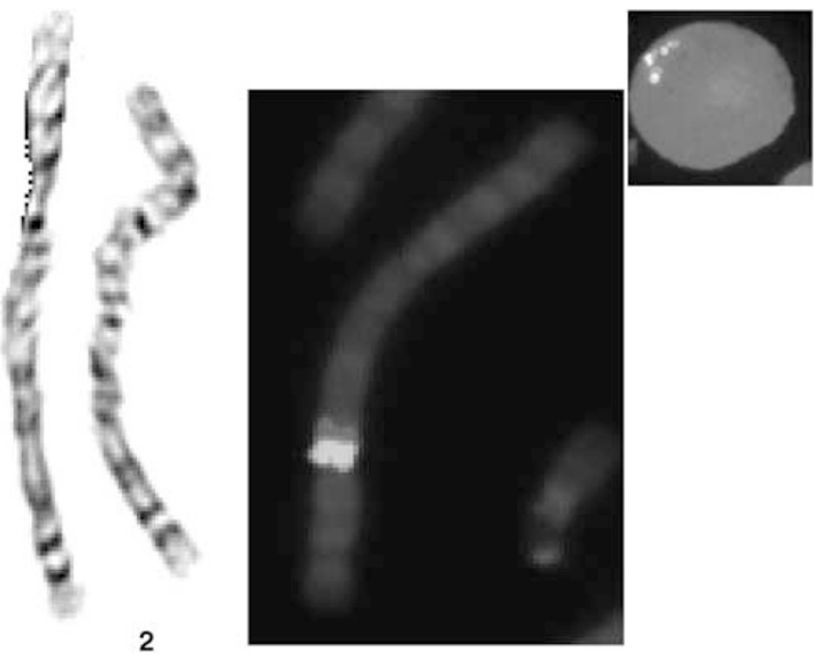

Figure 4 Karyotype and FISH analyses. Note tandem 2q duplication.

tyly, although further expression studies in cells from the developing autopod, rather than in lymphoblasts, would be needed to ascertain this. It has been suggested that the ELCR could be needed to 
implement colinear expression of the HOXD cluster. ${ }^{3}$ The duplication could disconnect the cluster from the ELCR and therefore explain the limb phenotype, although the recent report from Kantaputra et al ${ }^{11}$ did not identify the same $2 q$ duplication in other affected individuals and suggested that a balanced structural chromosomal rearrangement affecting HOXD locus regulation could also explain the phenotype.

A modification of the chromosomal environment due to the duplication could possibly be involved in the genesis of the finger phenotype. Such mechanisms have already been described by Dlugaszewska et $a l^{13}$ and correspond to translocations and inversions with breakpoints near the HOXD cluster. Patient 2 (as designated in the original article) carried a $\mathrm{t}(2 ; 10)(\mathrm{q} 31.1 ; \mathrm{q} 23.33)$ translocation with a proximal breakpoint around $1050 \mathrm{~kb}$ downstream to HOXD13. He harboured ulnar hypoplasia and absence of fingers 3 to 5 and hypoplastic fingers 1 and 2. In this case, a first wave impairment could be suspected because of the zeugopod involvement, and thus, ELCR misregulation might be involved in the phenotype. Regarding our patients, karyotype and FISH analyses allowed to confirm that the duplication was tandem, rather than being translocated to another chromosome.

It is known that HOXD products need to be adequately balanced for a normal digit pattern. Thus, we studied the expression of HOXD13, HOXD12 and HOXD10 in two affected patients carrying a $2 q 31.1 \mathrm{q} 31.2$ duplication. We showed that, in lymphobasts, the duplication was responsible for a complex modification of HOXD genes' expression, and we hypothesised that this may alter the limb bud development and cause the phenotype.

Indeed, the overexpression of the most $5^{\prime}$ located HOXD gene can, by itself, generate finger anomalies. It has been demonstrated that in presence of GLI3, HOXD10 upregulation induces polydactyly, whereas upregulation of HOXD13 and HOXD12 leads to oligodactyly. ${ }^{12}$ Moreover, the altered expression of HOXD genes probably modifies their pattern of expression and impairs the digit shaping as described in an animal model presenting an internal duplication of the complex. ${ }^{3,4}$ The overexpression of HOXD10 and HOXD13 modifies the ratio between $5^{\prime}$ HOXD genes and GLI3 products, probably mimicking a lack of GLI3 products that corresponds to Greig syndrome in which cutaneous syndactyly occurs.

For the genes presumptively involved in the nystagmus, $\mathrm{CHN} 1$ was overexpressed in the duplicated patients (2.1 fold in the father and 1.6 fold in the daughter), as well as CHRNA1 (1.3 fold in the father and 3.0 fold in the daughter; data not shown).

Ocular motility depends on the precise innervation of ocular motor muscles. Abnormal innervation can give rise to nystagmus. The CHN1 gene encodes two Rac-specific guanosine triphosphatase, activating $\alpha$-chimaerin isoforms. Miyake et al ${ }^{15}$ recently identified missense mutations in CHN1, which induce a gain-of-function of $\alpha 2$-chimaerin and cause aberrant innervation of oculomotor muscles in animal models. Thus, overexpression of this gene in our patients might cause hyperactivation of $\alpha$-chimaerin and impair normal ocular motor innervation, although Duane syndrome is distinct from nystagmus and we cannot prove this. The effect of this overexpression could possibly be modulated by the overexpression of CHRNA1. No other candidate gene seemed to be potentially associated with eye anomalies in the duplicated region. Another explanation could be the dysregulation of a gene, distant from the duplication, which we have not identified yet.

We show that duplication of the HOXD cluster disturbs, at least, HOXD10, HOXD12 and HOXD13 expression. This misregulation possibly gives rise to syndactyly through a direct effect of excessive HOXD genes' products, or because of ratio disequilibrium between $5^{\prime}$ HOXD and GLI3 products. In addition, the modification of chromosomal environment could be involved in the complex dysregulation. Further experiments in animal models are needed to confirm these hypotheses. Although CHN1 gene is the best candidate gene for the nystagmus, its overexpression might not be the only explanation for this finding.

\section{CONFLICT OF INTEREST}

The authors declare no conflict of interest.

1 Johnson RL, Tabin CJ: Molecular models for vertebrate limb development. Cell 1997; 90: 979-990.

2 Kessel M, Gruss P: Murine developmental control genes. Science 1990; 249: 374-379.

3 Zakany J, Kmita M, Duboule D: A dual role for Hox genes in limb anterior-posterior asymmetry. Science 2004; 304: 1669-1672.

4 Tarchini B, Duboule D: Control of Hoxd genes' collinearity during early limb development. Dev Cell 2006; 10: 93-103.

5 Goodman FR, Majewski F, Collins AL, Scambler PJ: A 117-kb microdeletion removing HOXD9-HOXD13 and EVX2 causes synpolydactyly. Am J Hum Genet 2002; 70: 547-555.

6 Del Campo M, Jones MC, Veraksa AN et al: Monodactylous limbs and abnormal genitalia are associated with hemizygosity for the human 2 q31 region that includes the HOXD cluster. Am J Hum Genet 1999; 65: 104-110.

7 Svensson AM, Curry CJ, South ST et al: Detection of a de novo interstitial 2q microdeletion by $\mathrm{CGH}$ microarray analysis in a patient with $\operatorname{limb}$ malformations, microcephaly and mental retardation. Am J Med Genet A 2007; 143A: 1348-1353.

8 Prontera P, Bernardini L, Stangoni $G$ et al: 2q31.2q32.3 deletion syndrome: report of an adult patient. Am J Med Genet A 2009; 149A: 706-712.

9 Pescucci C, Caselli R, Grosso S et al: 2q24-q31 deletion: report of a case and review of the literature. Eur J Med Genet 2007; 50: 21-32.

10 Boulet AM, Capecchi MR: Duplication of the Hoxd11 gene causes alterations in the axial and appendicular skeleton of the mouse. Dev Biol 2002; 249: 96-107.

11 Kantaputra PN, Klopocki E, Hennig BP et al: Mesomelic dysplasia Kantaputra type is associated with duplications of the HOXD locus on chromosome 2q. Eur J Hum Genet 2010; 18: 1310-1314.

12 Sheth R, Bastida MF, Ros M: Hoxd and Gli3 interactions modulate digit number in the amniote limb. Dev Biol 2007; 310: 430-441.

13 Dlugaszewska B, Silahtaroglu A, Menzel C et al: Breakpoints around the HOXD cluster result in various limb malformations. J Med Genet 2006; 43: 111-118.

14 Cho TJ, Kim OH, Choi IH et al: A dominant mesomelic dysplasia associated with a 1.0-Mb microduplication of HOXD gene cluster at 2q31.1. J Med Genet 2010; 47: 638-639.

15 Miyake N, Chilton J, Psatha M et al: Human CHN1 mutations hyperactivate alpha2chimaerin and cause Duane's retraction syndrome. Science 2008; 321: 839-843. 